\title{
Cuba's Scientific Contributions to Global Polio Eradication
}

\author{
Sonia Resik MD PhD, Ondrej Mach MD MPH, Alina Tejeda MD MS, Miguel A. Galindo MD, Roland W. Sutter MD MPH\&TM
}

\begin{abstract}
Cuba eliminated polio in 1962 and was among the first countries to do so. Since then, only 20 cases of vaccine-derived paralytic poliomyelitis have been reported. Because Cuba used oral poliovirus vaccine exclusively in two mass campaigns usually in February and April each year, Sabin viruses were detected only within approximately 6-8 weeks after each annual campaign. This made Cuba a very attractive site to study the epidemiology of poliomyelitis in a tropical country without risk of secondary transmission of Sabin viruses for a large
\end{abstract}

\section{INTRODUCTION}

In 1988, when the World Health Assembly resolved to eradicate poliomyelitis by the year 2000,[1] Cuba had achieved elimination over 25 years earlier and managed to remain polio free despite substantial travel by its population to polio-endemic countries. Two mass vaccination campaigns conducted in 1962, achieved very high coverage and eliminated wild poliovirus from Cuba.[2] In 1994, the entire Western Hemisphere was certified as polio free by PAHO's International Commission for the Certification of Eradication of Poliomyelitis.[3]

The rest of the world started to institute sensitive surveillance for acute flaccid paralysis, a clinical presentation that includes paralytic poliomyelitis, and started to plan for mass-campaign use of oral poliovirus vaccine (OPV) for eradication. Cuba and its Ministry of Public Health (MINSAP) expressed their willingness to contribute to these efforts by cooperating in expanding the scientific foundation for polio eradication and implementing related clinical trials and other evaluations.

Cuba already had an excellent health infrastructure for conducting scientific research, including clinical trials. Principal investigators, including the heads of the national poliovirus laboratory housed in the Pedro Kourí Tropical Medicine Institute (IPK), participated in such studies. IPK and its Ethics Committee reviewed and approved the clinical trials; while the Center for State Control of Medicines and Medical Devices approved vaccines for use in the studies, as well as study protocols. All trials were also approved by WHO's Ethics Review Board and the National Expanded Program on Immunization was also engaged. More recently, MINSAP established a field site for clinical trials with vaccines in Camagüey Province, in eastern Cuba. Thus, with MINSAP and Cuban government support, the necessary infrastructure was put in place for studies and clinical research jointly prioritized by MINSAP and WHO.

IMPORTANCE Collaboration between Cuba and WHO has been vital for the Global Polio Eradication Initiative. Cuban research findings over two decades have influenced global policy decision-making and made it possible to design innovative strategies for polio eradication. part of each year, an advantage over countries that used oral poliovirus vaccine continuously throughout the year in routine immunization programs. This report summarizes the unique scientific collaboration between Cuba's Ministry of Public Health and WHO, with participation by US scientists, in the global effort to eradicate polio.

KEYWORDS Poliomyelitis, disease eradication, disease elimination, oral poliovirus vaccine, Sabin vaccine, inactivated poliovirus vaccine, Salk vaccine, Cuba, WHO

This report summarizes the WHO-Cuban polio research collaboration, from the first scientific study concerning poliovirus eradication (1994) to the present. Many other studies that are part of this joint effort have been published previously.[4-16]

\section{SCIENTIFIC PROJECTS}

Cuba's part in the continuing collaboration with $\mathrm{WHO}$ has focused on issues relevant to policymaking for the ongoing global polio eradication effort. It has concentrated particularly on looking for answers to scientific questions that could not be answered elsewhere, taking into account Cuba's unique OPV vaccination program, carried out only twice annually, usually in February and April. Specifically, research has addressed several aspects of OPV and inactivated poliovirus vaccine (IPV), such as immunogenicity, adverse reactions and complications, persistence of Sabin virus in populations, the immunogenicity schedule and its affordability, number of doses needed, evaluation of new vaccines and devices, and booster response.

Studies completed and published OPV immunogenicity This study evaluated the seroprevalence of serotype-specific polio antibodies following 2, 4, and 6 OPV doses in Cuba. Results confirmed that 3 OPV doses were not sufficient to achieve high seroprevalence against all three serotypes in Cuba. This suggested that in other tropical zones, additional doses would be needed to ensure high population immunity.[4]

Assessment of intussusception risk after OPV This study was designed to address a concern that OPV could cause intussusception, which was found to be a problem with the first licensed rotavirus vaccine in the USA. No increased risk of intussusception after OPV vaccination was observed among Cuban infants,[5] alleviating concerns raised globally.

Vaccine-associated paralytic poliomyelitis (VAPP) In this study all cases of reported acute flaccid paralysis between 1963 and 2006 were analyzed to determine if they were associated with vaccine administration, after interruption of wild poliovirus circulation had been achieved in 1962. The study showed that VAPP risk was not elevated and was comparable to that reported in other countries.[6]

Sabin virus persistence in populations and the environment after mass OPV campaigns A series of studies attempted to characterize persistence of circulating Sabin virus after mass 
vaccination campaigns, using stool surveys, seroprevalence surveys and environmental sampling. Collectively, results demonstrated limited persistence, approximately 2-3 months, following a mass OPV campaign.[7,8] Finding such limited circulation, even in a tropical developing country, helped influence endgame policy development for WHO's global eradication program.[17]

IPV schedule immunogenicity This clinical trial assessed immunogenicity of a 2-dose IPV schedule (at 2 and 4 months) versus a 3 -dose schedule (at 6, 10 and 14 weeks). Both schedules provided similar seroconversion rates, $>80 \%$ for all three poliovirus serotypes. This study,[9] and one in Puerto Rico[18] demonstrated that immunogenicity of early IPV administration are greatly reduced by maternally derived antibody. Because the study included an unvaccinated control group, it was able to assess the contribution of two- and threedose IPV schedules on limiting poliovirus excretion after a trivalent OPV challenge dose, compared with the unvaccinated control arm. The results demonstrated no differences between groups in excretion prevalence seven days after challenge, confirming early reports from higher-income countries. However, the IPV group's virus titer was approximately 0.5 log10 lower than that of the control group.[9] The findings were subsequently confirmed in other studies in developing countries,[19-21] which demonstrated IPV does not induce mucosal immunity per se, but may accelerate development of such immunity, in line with other studies showing that IPV-vaccinated subjects excrete for shorter periods than nonvaccinated individuals.[22,23]

Affordable IPV This study, in conjunction with another in Oman,[24] evaluated the immunogenicity of fractional-dose IPV administered intradermally by needle-free jet injector (Biojector 2000). A fractional dose contains only one fifth of the IPV antigen contained in a full dose, enabling limited supplies to go farther. The study once more confirmed low immunogenicity of IPV when given in an early schedule (at 6, 10, and 14 weeks of age), suggesting maternally derived antibody interference with vaccine immune responses, highlighted by the large difference in immunogenicity between the fractional-dose group and the full-dose control group in this early schedule.[10] Nevertheless, it demonstrated feasibility of using needle-free jet injectors to administer IPV, and suggested that optimal immunogenicity may be achieved by starting IPV later in life and allowing a longer interval between doses.

Priming after IPV This clinical trial was an outcome of the previous study and addressed two questions. First, if the schedule starts later (here at four months) and the interval between doses is longer (here also four months) can the immunogenicity of a fractional- or a full-dose schedule be improved? Second, what does a single dose do in terms of immune response, defined as seroconversion and priming? The study demonstrated that 2 doses can seroconvert $>90 \%$ of vaccinees to all 3 serotypes with a 4 - and 8-month schedule. In addition, it elucidated for the first time the relative contribution of priming following a first dose of IPV. Although seroconversion to poliovirus type 2 was $47 \%$ and $63 \%$ following a fractional dose or full dose, respectively, of IPV, $>90 \%$ of vaccinees that did not seroconvert, responded with a priming immune response.[11] This study was instrumental in development of recommendations for a single dose of IPV following withdrawal of Sabin poliovirus type 2 from trivalent OPV and for an immunization schedule using two fractional doses. Subsequently, IPV priming was confirmed by studies in Bangladesh and in several Latin American countries.[18,19,25]

Devices for intradermal administration of fractional-dose IPV Several devices were evaluated to facilitate intradermal administration of fractional-dose IPV, including jet injectors: Biojector 2000 (Bioject, USA), Bioject ID Pen (Bioject, USA), Tropis (Pharmajet, USA). These studies compared devices' usability and ability to induce immune response with administration by BCG needle and syringe, and full-dose IPV administered intramuscularly. Three dimensions were assessed: immune response indicated by seroconversion or by increase in antibody titers; quality of injection (bleb formation, liquid leaking); and vaccinators' and parents' preferences for administration method. These evaluations demonstrated that, with one exception, ability to induce immune response with the devices was comparable to that using BCG needle and syringe administration. There was no clear association between immune response and bleb size or quantity of liquid leaking from dermal injection sites. Ergonomic assessment stimulated further engineering improvements of at least one device.[12,13] As reported previously, health care providers and parents preferred needle-free devices by an overwhelming majority.[13,24]

New vaccine evaluation (IPV produced from Sabin strains) This study evaluated immunogenicity and reactogenicity of two formulations of Sabin IPV, a plain and an adjuvanted vaccine, with conventional Salk IPV as a control. Between $90 \%$ and $100 \%$ of subjects responded with either seroconversion or a 4-fold boosting of antibody titers. No serious adverse events were reported during the six-month followup period.[14] A followup study evaluated antibody decay 21-22 months after initial vaccination and reported that it was similar in all 3 study groups.[15] These studies provided further confidence in Sabin IPV development efforts.

Booster response following fractional-dose IPV This study was designed to demonstrate noninferiority of fractional-dose compared with full-dose IPV. There were no significant differences in booster response 7, 28 and 56 days after a first or second dose vaccination. The study concluded that fractional-dose IPV induced a booster immune response similar to that of full-dose IPV, and that fractional-dose IPV could stretch available supplies when needed in an outbreak response situation.[16] The earlier fractional-dose study[11] and followup studies provided the scientific basis for recommending a fractional-dose IPV schedule. $[26,27]$

Studies in progress Several studies and evaluations are continuing and are expected to yield important new findings to further guide policy decision-making for the Global Polio Eradication Initiative. These studies will help clarify the role of IPV in mucosal immunity and provide information on intramuscular fractional-dose IPV, specifically:

- mucosal immunity following IPV,

- cross-immunity following bivalent OPV and IPV versus IPV alone,

- disappearance of poliovirus type 2 in sewage after the switch from trivalent to bivalent OPV, 
- intramuscular fractional-dose IPV given at 4 and 8 months, and

- pilot introduction of one needle-free device to administer fractional-dose IPV. This pilot will help guide policy decisions for such devices, especially in Cuba.

\section{LOOKING FORWARD}

Cuba has proven a useful resource and partner for polio eradication, acting as an incubator for testing new ideas and approaches. The
WHO-Cuban collaboration, lasting for over 20 years, has been vital for the Global Polio Eradication Initiative, making it possible to design innovative strategies, especially for the polio eradication endgame[17] and for immunization policy development worldwide. [28] Collectively, MINSAP and the global community need to ensure that this resource, including associated infrastructure, is protected, in order to be able to conduct clinical trials that meet and surpass the highest ethical and scientific standards. $-1 /$ -

\section{REFERENCES}

1. World Health Organization. WHA41.28. Global eradication of poliomyelitis by the year 2000 Geneva: World Health Organization; 1988 May. 1 p.

2. Rodríguez Cruz R. Cuba: mass polio vaccination program, 1962-1982. Rev Infect Dis. 1984 MayJun;6 Suppl 2:S408-12.

3. Robbins FC, de Quadros CA. Certification of the eradication of indigenous transmission of wild poliovirus in the Americas. J Infect Dis. 1997 Feb;175 Suppl 1:S281-5.

4. Más Lago $P$, Ramón Bravo J, Andrus JK, Comellas MM, Galindo MA, de Quadros CA, et al. Lessons from Cuba: mass campaign administration of trivalent oral poliovirus vaccine and seroprevalence of poliovirus neutralizing antibodies. Bull World Health Organ. 1994;72(2):221-5.

5. Sardiñas MA, Cárdenas $A Z$, Coutin GM, Peña MS, Santiago MA, Sánchez MV, et al. Lack of association between intussusception and oral polio vaccine in Cuban children. Eur J Epidemiol. 2001;17(8):783-7.

6. Más-Lago P, Ferrer H, Goyenechea Á, Galindo Á, Sarmiento L, Fonseca M, et al. Casos de poliomielitis paralítica asociada a la vacuna oral antipoliomielítica en Cuba (1963-2006). Rev Cubana Hig Epidemiol. 2008 May-Aug;46:2. Spanish.

7. Más Lago P, Cáceres VM, Galindo MA, Gary $\mathrm{HE}$ Jr, Valcarcel M, Barrios J, et al. Persistence of vaccine-derived poliovirus following a mass vaccination campaign in Cuba: implications for stopping polio vaccination after global eradication. Int J Epidemiol. 2001 Oct;30(5):1029-34.

8. Más Lago P, Gary HE Jr, Pérez LS, Cáceres V, Olivera JB, Puentes RP, et al. Poliovirus detection in wastewater and stools following an immunization campaign in Havana, Cuba. Int J Epidemiol. 2003 Oct;32(5):772-7.

9. Cuba IPV Study Collaborative Group. Randomized, placebo-controlled trial of inactivated poliovirus vaccine in Cuba. $\mathrm{N}$ Engl $\mathrm{J}$ Med. 2007 Apr 12;356(15):1536-44.

10. Resik S, Tejeda A, Lago PM, Díaz M, Carmenates $A$, Sarmiento $L$, et al. Randomized controlled clinical trial of fractional doses of inactivated poliovirus vaccine administered intradermally by needle-free device in Cuba. J Infect Dis. 2010 May 1;201(9):1344-52.

11. Resik S, Tejeda A, Sutter RW, Díaz M, Sarmiento $\mathrm{L}$, Alemañi N, et al. Priming after a fractional dose of inactivated poliovirus vaccine. $\mathrm{N}$ Engl J Med. 2013 Jan 31;368(5):416-24.

12. Resik S, Tejeda A, Mach O, Fonseca M, Díaz $\mathrm{M}$, Alemany $\mathrm{N}$, et al. Immune responses after fractional doses of inactivated poliovirus vaccine using newly developed intradermal jet injectors: a randomized controlled trial in Cuba. Vaccine. 2015 Jan 3;33(2):307-13.

13. Resik S, Tejeda A, Mach O, Sein C, Molodecky $\mathrm{N}$, Jarrahian $\mathrm{C}$, et al. Needle-free jet injector intradermal delivery of fractional dose inactivated poliovirus vaccine: Association between injection quality and immunogenicity. Vaccine. 2015 Oct 26;33(43):5873-7.
14. Resik S, Tejeda A, Fonseca M, Alemañi $N$ Díaz $M$, Martínez $Y$, et al. Reactogenicity and immunogenicity of inactivated poliovirus vaccine produced from Sabin strains: a phase I Trial in healthy adults in Cuba. Vaccine. 2014 Sep 22;32(42):5399-404.

15. Resik S, Tejeda A, Fonseca M, Sein C, Hung LH, Martínez Y, et al. Decay of Sabin inactivated poliovirus vaccine (IPV)-boosted poliovirus antibodies. Trials Vaccinol. 2015;4:71-4.

16. Resik S, Tejeda A, Díaz M, Okayasu H, Sein $C$, Molodecky NA, et al. Boosting immune responses following fractional-dose inactivated poliovirus vaccine: a randomized, controlled trial J Infect Dis. 2017 Jan 15;215(2):175-82.

17. World Health Organization. Global Polio Eradication Initiative. Polio Eradication \& Endgame Strategic Plan 2013-2018. Geneva: World Health Organization; CDC; UNICEF; 2013. $124 \mathrm{p}$.

18. Dayan $\mathrm{GH}$, Thorley $\mathrm{M}$, Yamamura $\mathrm{Y}$, Rodríguez $\mathrm{N}$, McLaughlin S, Torres LM, et al. Serologic response to inactivated poliovirus vaccine: a randomized clinical trial comparing 2 vaccination schedules in Puerto Rico. J Infect Dis. 2007 Jan 1;195(1):12-20.

19. Anand A, Zaman K, Estívariz CF, Yunus M, Gary HE, Weldon WC, et al. Early priming with inactivated poliovirus vaccine (IPV) and intradermal fractional dose IPV administered by a microneedle device: A randomized controlled trial. Vaccine. 2015 Nov 27:33(48):6816-22.

20. O'Ryan M, Bandyopadhyay AS, Villena R, Espinoza M, Novoa J, Weldon WC, et al. Chilean IPV/bOPV study group. Inactivated poliovirus vaccine given alone or in a sequential schedule with bivalent oral poliovirus vaccine in Chilean infants: a randomised, controlled, open-label, phase 4 , non-inferiority study. Lancet Infect Dis. 2015 Nov;15(11):1273-82.

21. Sutter RW, Bahl S, Deshpande JM, Verma H, Ahmad M, Venugopal P, et al. Immunogenicity of a new routine vaccination schedule for global poliomyelitis prevention: an open-label, randomised controlled trial. Lancet. 2015 Dec 12;386(10011):2413-21.

22. Ghendon $Y Z$, Sanakoyeva II. Comparison of the resistance of the intestinal tract to poliomyelitis vaccine (Sabin strains) in persons after naturally and experimentally acquired immunity. Acta Virol. 1961;5(5):265-73.

23. Collett MS, Hincks JR, Benschop K, Duizer $\mathrm{E}$, van der Avoort $\mathrm{H}$, Rhoden $\mathrm{E}$, et al. Antiviral activity of pocapavir in a randomized, blinded, placebo-controlled human oral poliovirus vaccine challenge model. J Infect Dis. 2017 Feb 1;215(3):335-43.

24. Mohammed AJ, AIAwaidy S, Bawikar S, Kurup PJ, Elamir E, Shaban MM, et al. Fractional doses of inactivated poliovirus vaccine in Oman. N Engl J Med. 2010 Jun 24;362(25):2351-9.

25. Asturias EJ, Bandyopadhyay AS, Self S, Rivera L, Saez-Llorens X, López E, et al. Latin American IPV001BMG Study Group. Humoral and intestina immunity induced by new schedules of bivalent oral poliovirus vaccine and one or two doses of inactivated poliovirus vaccine in Latin American infants: an open-label randomised controlled trial. Lancet. 2016 Jul 9;388(10040):158-69.

26. Anand A, Molodecky NA, Pallansch MA, Sutter RW. Immunogenicity to poliovirus type 2 following two doses of fractional intradermal inactivated poliovirus vaccine: a novel dose sparing immunization schedule. Vaccine. 2017 May 19;35(22):2993-8.

27. Okayasu H, Sein C, Chang Blanc D, González $A R$, Zehrung D, Jarrahian $C$, et al. Intradermal administration of fractional doses of inactivated poliovirus vaccine: a dose-sparing option for polio immunization. J Infect Dis. 2017 Jul 1;216(Suppl 1):S161-7.

28. World Health Organization. Meeting of the Strategic Advisory Group of Experts on immunization, October 2016 - conclusions and recommendations. Wkly Epidemiol Rec. 2016 Dec 2;91(48):561-82.

\section{THE AUTHORS}

Sonia Resik, physician with a master's degree in virology and a doctorate in medical sciences. Senior professor, senior researcher and Director, National Polio Laboratory Network, Pedro Kourí Tropical Medicine Institute, Havana, Cuba.

Ondrej Mach, physician specializing in epidemiology. Technical Officer, Research, Policy and Containment, Polio Eradication Department, WHO, Geneva, Switzerland.

Alina Tejeda, physician specializing in epidemiology, with a master's degree in infectious diseases. Epidemiologist, Provincial Health Office, Camagüey, Cuba.

Miguel A. Galindo, physician specializing in epidemiology. Immunization Advisor, Ministry of Public Health, Havana, Cuba.

Roland W. Sutter (Corresponding author: sutterr@who.int), physician specializing in epidemiology. CDC epidemiologist during part of the study period; Coordinator, Research, Policy and Containment, Polio Eradication Department, WHO, Geneva, Switzerland.

Submitted: October 3, 2017

Approved for publication: March 18, 2018 Disclosures: None 